\title{
FE DE ERRORES
}

\section{Fe de errores: Frecuencia de los tipos de infarto agudo de miocardio según la tercera definición [Rev Colomb Cardiol. 2017;24(6):592-597]}

\section{Erratum: Frequency of myocardial infarction according to the third universal definition [Rev Colomb Cardiol. 2017;24(6):592-597]}

\section{Paola Calvachi Prieto ${ }^{a, b}$, Dormar David Barrios ${ }^{c}$, Mariana Puccini ${ }^{b}$, Henry Alberto Mojica ${ }^{b}$, David Delgadillo ${ }^{a, b}$, Mabel Gómez $^{a, c}$, Edgar Celis $^{a}$ y Andrés Felipe Buitrago ${ }^{a, c, *}$}

\footnotetext{
a Departamento de Medicina Crítica y Cuidado Intensivo, Hospital Universitario Fundación Santa Fe de Bogotá, Bogotá, Colombia b Universidad de los Andes, Facultad de Medicina, Bogotá, Colombia

c Departamento de Medicina Interna, sección de Cardiología, Hospital Universitario Fundación Santa Fe de Bogotá, Bogotá, Colombia
}

Recibido el 12 de enero de 2018; aceptado el 15 de enero de 2018

En el artículo publicado anteriormente, se ha detectado un error en la filiación de uno de los autores. La filiación correcta del autor Dormar David Barrios ${ }^{\mathrm{c}, \mathrm{d}}$ es la siguiente:

c Departamento de Medicina Interna, sección de Cardiología, Hospital Universitario Fundación Santa Fe de Bogotá, Bogotá, Colombia

' Escuela de Medicina y Ciencias de la Salud, Universidad del Rosario, Bogotá, Colombia

Véase contenido relacionado en DOI: https://doi.org/10.1016/ j.rccar.2017.06.008

* Autor para correspondencia.

Correos electrónicos: gnanclares@osakidetza.net, abuitrag@uniandes.edu.co (A.F. Buitrago). 